\title{
MnTBAP therapy attenuates the downregulation of sodium transporters in obstructive kidney disease
}

\author{
Mi Liu ${ }^{1,2}$, Yangyang Zhu ${ }^{1,4}$, Ying Sun ${ }^{1,4}$, Zhaoying Wen ${ }^{5}$, Songming Huang ${ }^{1,3}$, Guixia \\ Ding ${ }^{1,3}$, Aihua Zhang ${ }^{1,3}$, Zhanjun Jia ${ }^{1,3}$ and Yue Zhang ${ }^{1,3}$ \\ ${ }^{1}$ Nanjing Key Laboratory of Pediatrics, Children's Hospital of Nanjing Medical University, Nanjing, China \\ ${ }^{2}$ Department of Cardiology, Sun Yat-sen Memorial Hospital, Sun Yat-sen University, Guangzhou, China \\ ${ }^{3}$ Department of Nephrology, Children's Hospital of Nanjing Medical University, Nanjing, China \\ ${ }^{4}$ Jiangsu Key Laboratory of New Drug Research and Clinical Pharmacy, Xuzhou Medical University, Xuzhou, China \\ ${ }^{5}$ Department of Radiology, Beijing Anzhen Hospital, Capital Medical University, Beijing Institute of Heart, Lung and Blood \\ Vessel Diseases, Beijing, China \\ Correspondence to: Zhanjun Jia, email: jiazj72@hotmail.com \\ Yue Zhang, email: zyflora2006@hotmail.com
}

Keywords: unilateral ureteral obstruction; mitochondrial oxidative stress; sodium transporters; MnTBAP; SOD

Received: July 18, $2017 \quad$ Accepted: November 13, $2017 \quad$ Published: December 07, 2017

Copyright: Liu et al. This is an open-access article distributed under the terms of the Creative Commons Attribution License 3.0 (CC BY 3.0), which permits unrestricted use, distribution, and reproduction in any medium, provided the original author and source are credited.

\section{ABSTRACT}

Ureteral obstruction is associated with reduced expressions of renal sodium transporters, which contributes to impaired urinary concentrating capacity. In this study, we employed a synthetic mitochondrial superoxide dismutase 2 (SOD2) mimic MnTBAP to investigate the role of mitochondrial oxidative stress in modulating the sodium transporters in obstructive kidney disease. Following unilateral ureteral obstruction (UUO) for 7 days, a global reduction of sodium transporters including NHE3, NCC, NKCC2, and ENaCa was observed as determined by qRT-PCR, Western Blotting or immunohistochemistry. Among these sodium transporters, the downregulation of NHE3, NCC, and NKCC2 was partially reversed by MnTBAP treatment. In contrast, the reduction of ENaCa was not affected by MnTBAP. The $\beta$ and $y$ subunits of ENaC were not significantly altered by ureteral obstruction or MnTBAP therapy. To further confirm the anti-oxidant effect of MnTBAP, we examined the levels of TBARs in the urine collected from the obstructed ureters of UUO mice and bladder of sham mice. As expected, the increment of urinary TBARs in UUO mice was entirely abolished by MnTBAP therapy, indicating an amelioration of oxidative stress. Meantime, we found that three types of SOD were all reduced in obstructed kidneys determined by qRTPCR, which was unaffected by MnTBAP. Collectively, these results demonstrated an important role of mitochondrial oxidative stress in mediating the downregulation of sodium transporters in obstructive kidney disease.

\section{INTRODUCTION}

Unilateral ureteral obstruction (UUO) is a wellestablished experimental rodent model that mimics the complex pathophysiology of chronic obstructive nephropathy in an accelerated manner. Release of obstruction is often associated with diuresis or natriuresis, which is related to the downregulation of water channels and sodium transporters in obstructed kidneys [1]. It has been demonstrated that both Angiotensin (Ang) II [2] and cyclooxygenase-2 (COX-2)derived prostaglandin E2 (PGE2) [3] mediated the decrease of sodium transporters in obstructive kidneys. However, the mechanisms mediating the downregulation of sodium transporters remain incompletely understood. 
Oxidative stress contributes importantly to the pathogenesis of UUO [4-6]. ROS production in the kidney is derived from multiple intracellular mechanisms including xanthine oxidase, cytochrome P450 systems, uncoupled Nitric Oxide synthase (NOS), mitochondrial respiratory chain and nicotinamide adenine dinucleotide phosphate (NADPH) oxidase (NOXs) [7-9]. It has been shown that oxidative stress in UUO contributes to the development of tubulointerstitial lesions and renal fibrosis [7-9]. Liu et al. demonstrated that NOX-derived ROS are partially responsible for the downregulation of renal tubular $\mathrm{Na} / \mathrm{K}$-ATPase expression in acute UUO rats [10]. Our group reported that inhibition of mitochondrial complex-1 also reversed the downregulation of aquaporins [11] and sodium transporters including NKCC2 and $\mathrm{ENaC} \alpha$ [12] in obstructive nephropathy. However, whether mitochondria-derived ROS is involved in the downregulation of sodium transporters during kidney obstruction is not known.

Therefore, in the present study, we employed manganese (III) tetrakis (4-benzoic acid) porphyrin chloride (MnTBAP), a synthetic mitochondrial superoxide dismutase 2 (SOD2) mimic, to investigate whether mitochondrial ROS could affect the downregulation of sodium transporters in the obstructed kidneys.

\section{RESULTS}

\section{Effects of MnTBAP therapy on the protein expressions of NHE3 and NCC in obstructed kidneys}

To detect the effect of MnTBAP therapy on the expression of sodium transporters in obstructed kidneys, we firstly examined the protein levels of NHE3 and NCC using Western blotting. As shown in Figure $1 \mathrm{~A}-1 \mathrm{C}$, both NHE3 and NCC protein expression were robustly downregulated in obstructive kidneys while MnTBAP therapy resulted in partially but significantly reversed the downregulation of NHE3 and NCC. These data indicated that mitochondrial oxidative stress was possibly involved in the downregulation of NHE3 and NCC in obstructed kidneys.

\section{Effect of MnTBAP therapy on the protein expression of $\mathrm{NKCC} 2$ in obstructed kidneys}

Next, we detected the effect of MnTBAP therapy on the protein expression of NKCC2. Consistent with previous reports $[1,12,13]$, NKCC2 protein expression in obstructed kidneys was dramatically decreased. Upon administration of MnTBAP, the reduction was significantly ameliorated (Figure 2A, 2B). By immunochemistry, we further confirmed the change of NKCC2 in obstructed kidneys with or without MnTBAP treatment (Figure 3). These data also suggested an important role of mitochondrial ROS in mediating the downregulation of NKCC2 in the obstructed kidneys.

\section{Effects of MnTBAP on the protein expressions of ENaC subunits in obstructed kidneys}

The epithelial sodium channel(ENaC) is expressed in the aldosterone sensitive distal nephron(ASDN) and consists of 3 subunits. In previous studies, we found distinct changes of three $\mathrm{ENaC}$ subunits in obstructed kidneys [12]. Here, similar to the sodium transporters described above, the protein level of $\mathrm{ENaC} \alpha$ displayed a marked downregulation in obstructed kidneys. However, MnTBAP administration did not affect the reduction of $\mathrm{ENaC} \alpha$ in obstructed kidneys (Figure 4A, 4B). For $\mathrm{ENaC} \gamma$, we observed a trend of downregulation in obstructed kidneys, which was not altered by MnTBAP therapy (Figure 4A, 4C). These results suggested that the alteration of ENaC subunits in obstructed kidneys is possibly independent of mitochondrial oxidative stress.

\section{Effects of MnTBAP on the mRNA expressions of sodium transporters in obstructed kidneys}

To clarify whether MnTBAP therapy affected the regulation of sodium transporters at a transcriptional level, we analyzed the mRNA expressions of individual transporters via qRT-PCR. In obstructed kidneys, NHE3, NCC, NKCC2, and ENaC $\alpha$ were all significantly downregulated (Figure 5A-5D). However, none of the decreased transporters was influenced by MnTBAP therapy (Figure 5A-5D). These results implied that mitochondrial oxidative stress might be involved in the downregulation of the protein levels of sodium transporters along the nephron except ENaCs via a posttranscriptional mechanism. Thus, inhibition of the mitochondrial oxidative stress by MnTBAP couldn't rescue the downregulation of sodium transporters at a transcriptional level, leading to a partial effect of MnTBAP therapy on the reduced sodium transporters of NHE3, NCC, and NKCC2.

\section{Effects of MnTBAP on the expressions of SODs and oxidative stress in obstructed kidneys}

As a mitochondrial SOD mimics, MnTBAP showed a protective role against the downregulation of sodium transporters in obstructed kidneys, suggesting a possible defect of SOD-related antioxidant system. To validate the anti-oxidative effect of MnTBAP in this model, we measured the urinary levels of TBARS, a known marker of oxidative stress, using the urine collected from the obstructed ureters of UUO mice and bladder of sham mice. As shown in Figure 6, MnTBAP therapy completely normalized the increment of TBARS concentration in urine. Furthermore, we analyzed the profiles of SODs 
A

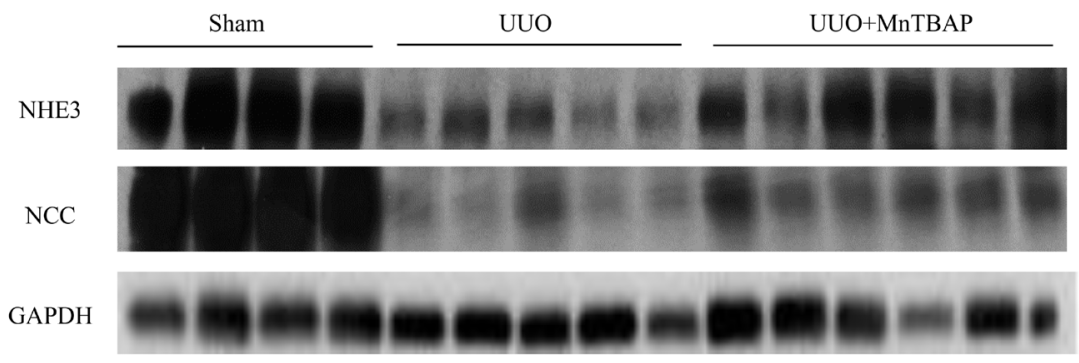

B

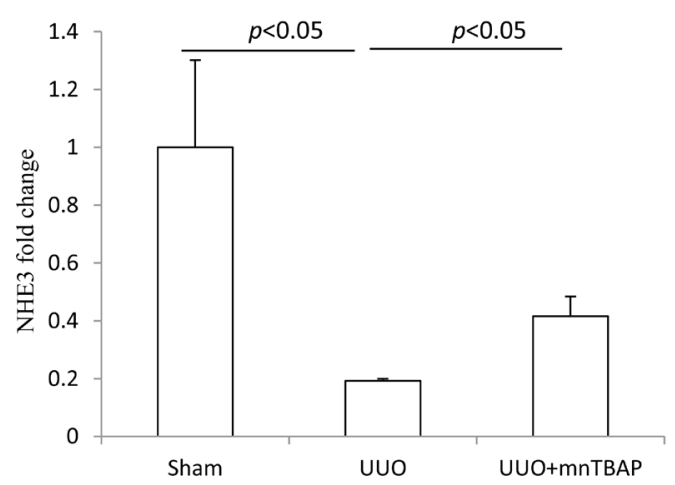

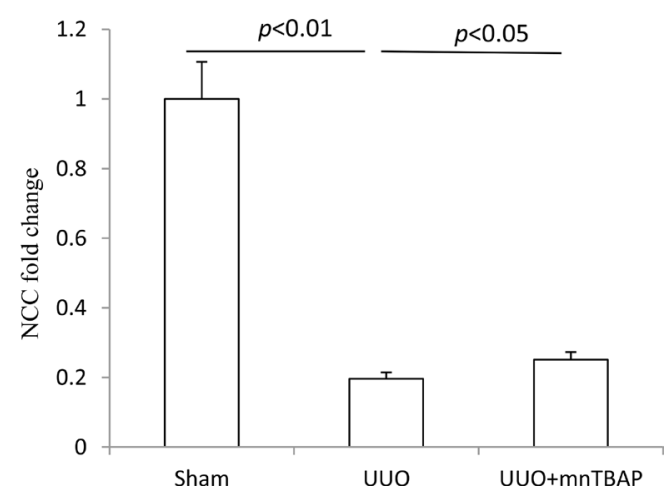

Figure 1: Protein expressions of NHE3 and NCC in obstructed kidneys following MnTBAP treatment. (A) Western blotting analysis of NHE3 and NCC. (B) Densitometric analysis of NHE3 normalized by GAPDH. (C) Densitometric analysis of NCC normalized by GAPDH. The presented data are means \pm SE. $N=6$ in each group.

A

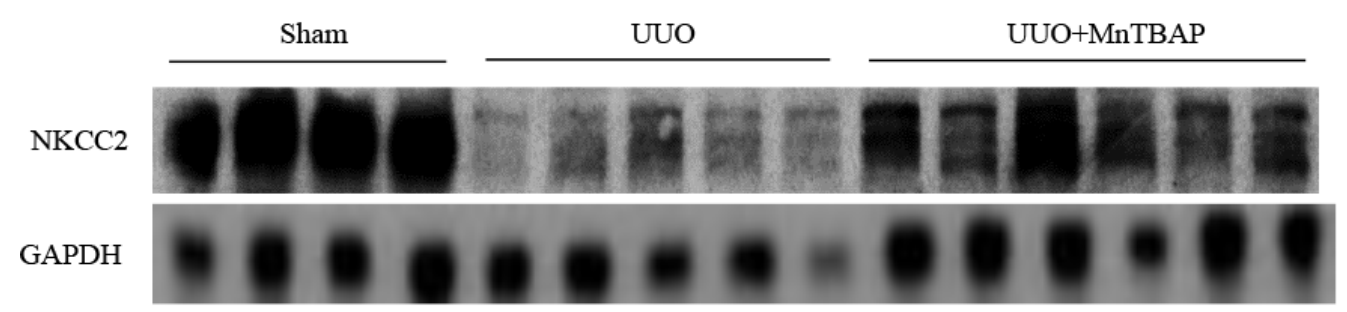

B

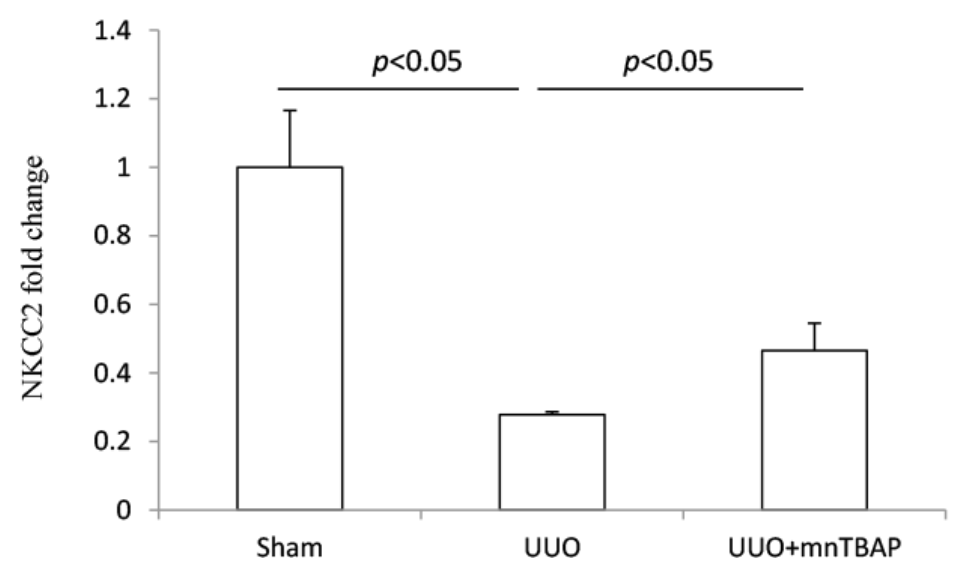

Figure 2: Protein expression of NKCC2 in obstructed kidneys following MnTBAP treatment. (A) Western blotting analysis of NKCC2. (B) Densitometric analysis of NKCC2 normalized by GAPDH. The presented data are means \pm SE. $N=6$ in each group. 

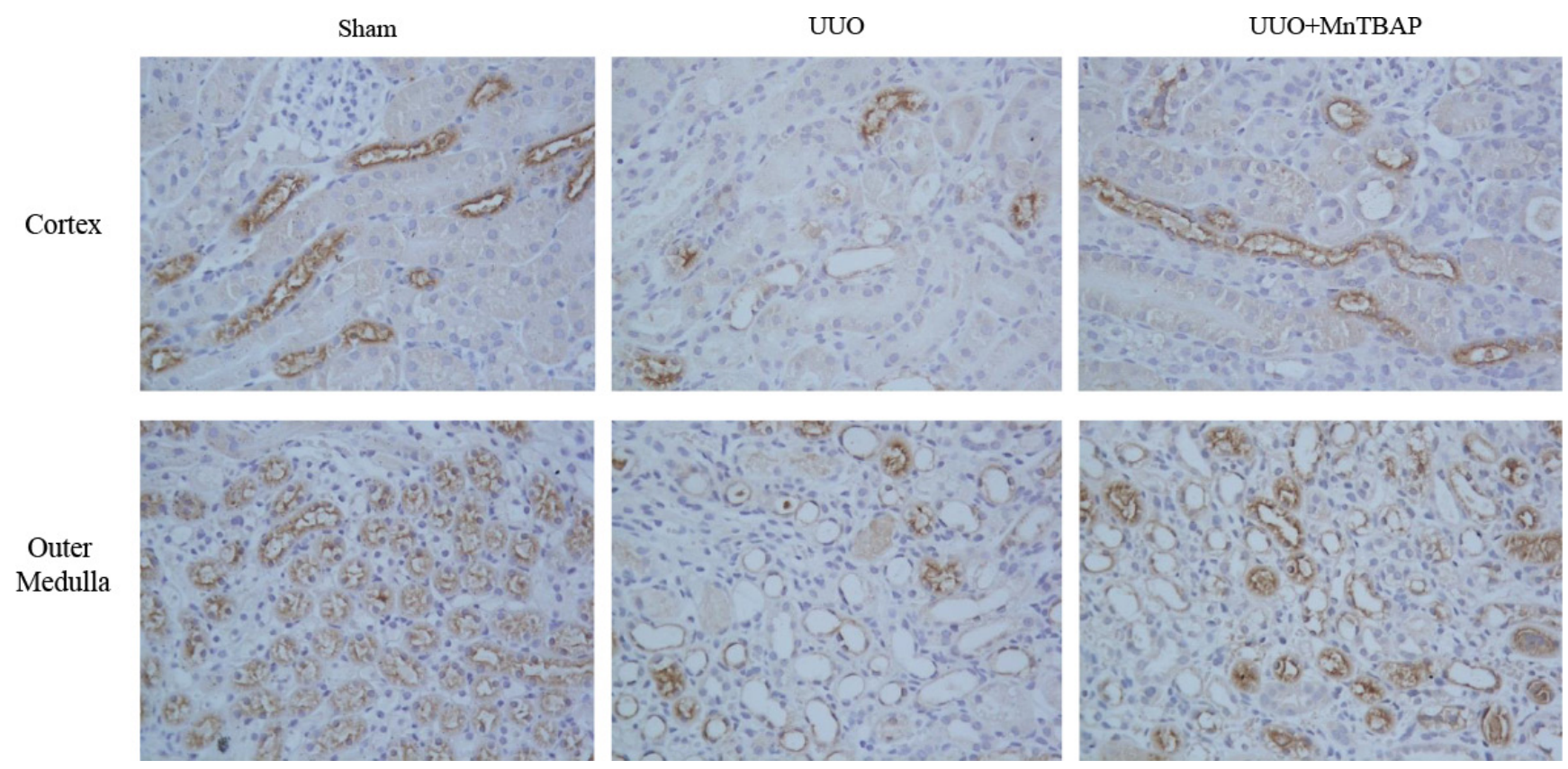

Figure 3: Immunohistochemistry of NKCC2 in obstructed kidneys following MnTBAP treatment.

$\mathbf{A}$

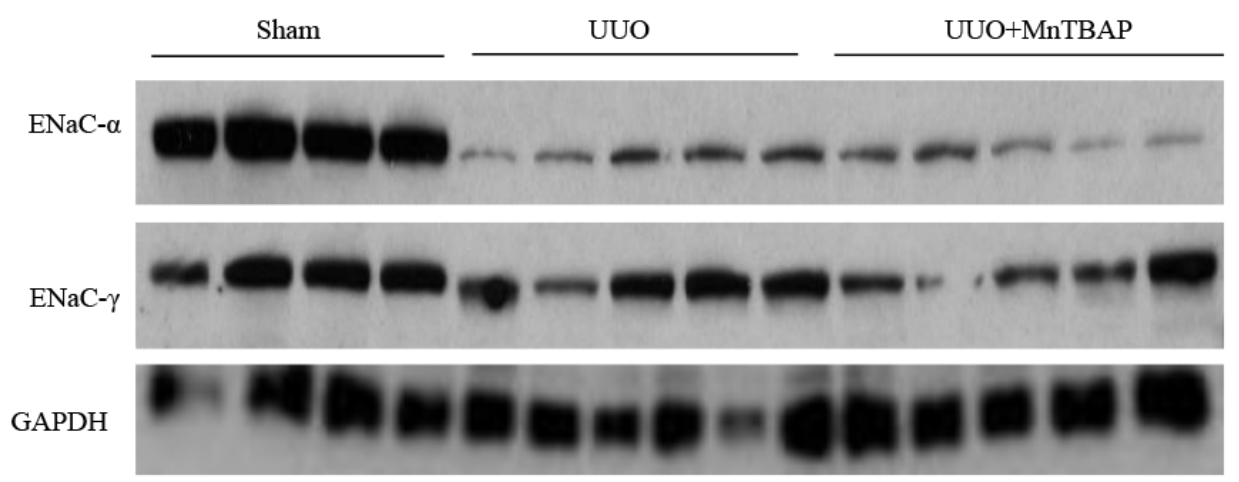

B

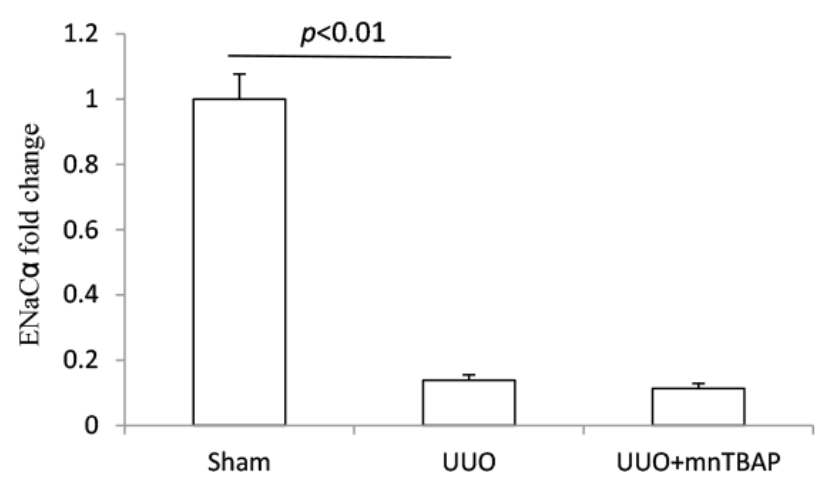

C

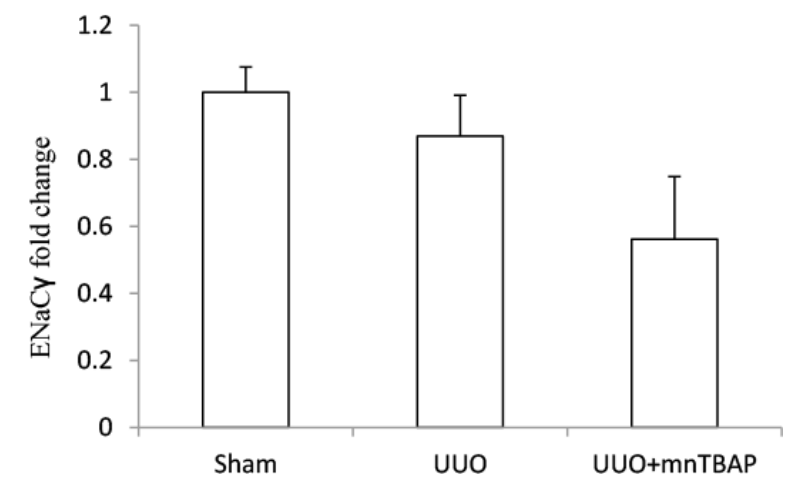

Figure 4: Protein expressions of ENaCa and ENaC $\gamma$ in obstructed kidneys following MnTBAP treatment. (A) Western blotting analysis of $\mathrm{ENaC} \alpha$ and $\mathrm{ENaC} \gamma$. (B) Densitometric analysis of $\mathrm{ENaC} \alpha$ normalized by GAPDH. (C) Densitometric analysis of $\mathrm{ENaC} \gamma$ normalized by GAPDH. The presented data are means \pm SE. $N=6$ in each group. 
(SOD1, SOD2, and SOD3) by qRT-PCR. Notably, all three forms of SODs were significantly downregulated in obstructed kidneys, which was not affected by MnTBAP therapy (Figure 7A-7C). These results suggested a potent role of MnTBAP in antagonizing the oxidative stress in obstructive kidneys. Finally, we detected the morphological changes using a PAS staining and found that MnTBAP treatment did not significantly affected the tubular structure in obstructed kidneys (Figure 8).

\section{DISCUSSION}

Urinary tract obstruction is a serious disorder accompanied with impaired renal function such as a compromised ability to regulate the urinary excretion of water and sodium. It has been well documented that the abundance of sodium transporters was significantly reduced in response to ureteral obstruction, which at least partially contribute to the impaired urine concentrating capacity after the release of urinary tract obstruction [1, 13-15]. Consistent with previous finding, our data showed that the renal expression of sodium transporters including NHE3, NCC, NKCC2, and $\mathrm{ENaC} \alpha$ were robustly decreased after ureteral obstruction while the expression of $\mathrm{ENaC} \gamma$ was not significantly changed in obstructed kidneys. Unlike other sodium transporters, the distinct regulation of $\mathrm{ENaC}$ subunits was reported previously by our and other groups $[12,16,17]$.
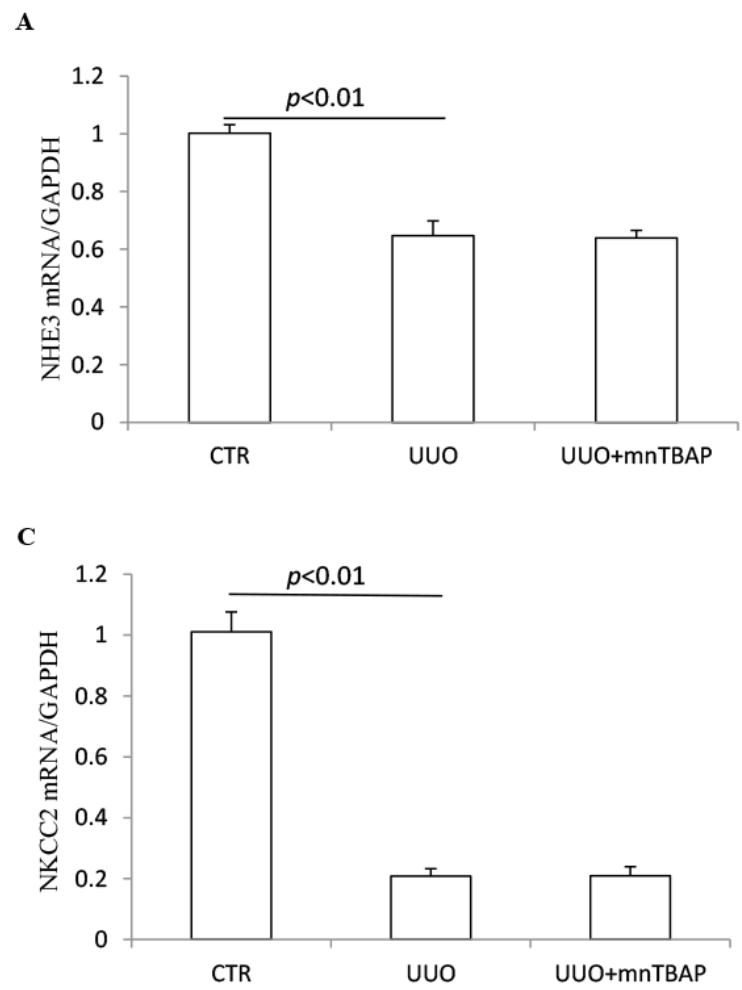

Oxidative stress was well described to contribute importantly to the pathogenesis of obstructive nephropathy $[6,18-22]$. The hydrostatic pressure and ischemia decreased intrarenal oxygen tension and resulted in increased oxidative stress. Various ROS sources implicated in renal diseases include the mitochondrial respiratory chain [23], NADPH oxidase [24, 25], xanthine oxidase [26], cyclooxygenase [27], lipoxygenase and uncoupled nitric oxide synthase (NOS) [28]. Among them, mitochondria are regarded as the major source of ROS since they are the primary intracellular sites of oxygen consumption. Xu et al. demonstrated that mitochondrial oxidative stress was associated with autophagy and apoptosis in tubular cells and represented an important mechanism of tubular decomposition and nephron loss during UUO [29]. Nishida's group reported that mitochondrial dysfunction led to an elevation of ROS, which resulted in kidney fibrosis by stimulating cellular transformation to myofibroblasts during obstructive nephropathy [30]. Moreover, Manucha et al. reviewed that there existed an interaction among mitochondrial oxidative stress, apoptosis and inflammation during obstructive nephropathy [19]. Our group further confirmed this interaction by showing that mitochondrial complex I inhibitor rotenone attenuated not only oxidative stress but also inflammation and fibrosis in chronic obstructive uropathy [31]. At the meantime, we observed attenuated downregulation of sodium transporters by rotenone

B

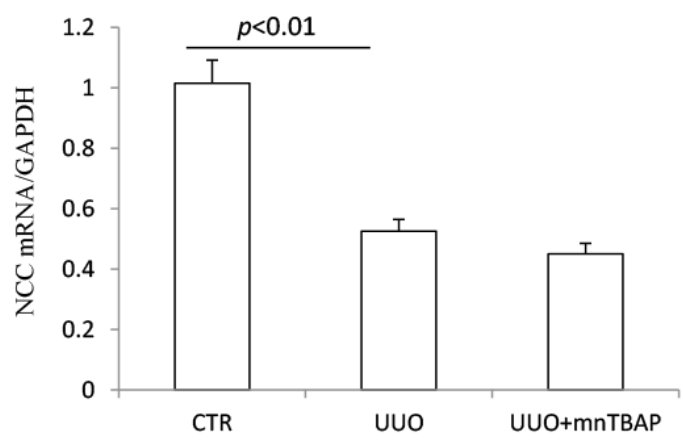

D

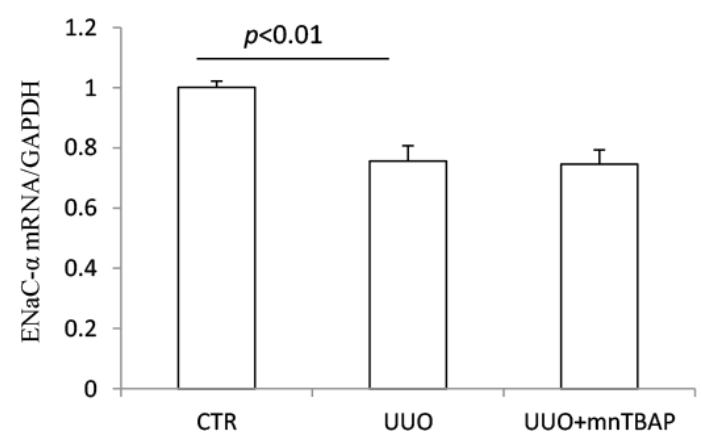

Figure 5: mRNA expressions of sodium transporters in obstructed kidneys following MnTBAP treatment. (A) qRT-PCR analysis of NHE3. (B) qRT-PCR analysis of NCC. (C) qRT-PCR analysis of NKCC2. (D) qRT-PCR analysis of ENaCa. The presented data are means \pm SE. $N=6$ in each group. 
in UUO mice, which indicated the involvement of mitochondria dysfunction in defective sodium handling in obstructed kidney. Mitochondrial dysfunction results in ATP depletion, reactive oxygen species (ROS) overproduction, and the release of proapoptotic factors such as cytochrome $\mathrm{C}$ and mitochondrial DNA. The known role of mitochondria-localized SOD2 in antagonizing oxidative stress gave us a clue that mitochondrial oxidative stress might contributed to the dysregulation of sodium transporter during UUO. The superoxide dismutase (SOD) family is the main antioxidant system and is of importance in oxidative stress modulation [32]. Increased oxidative stress in obstructive nephropathy is always accompanied by decreased SOD expression [5, 33]. Our results

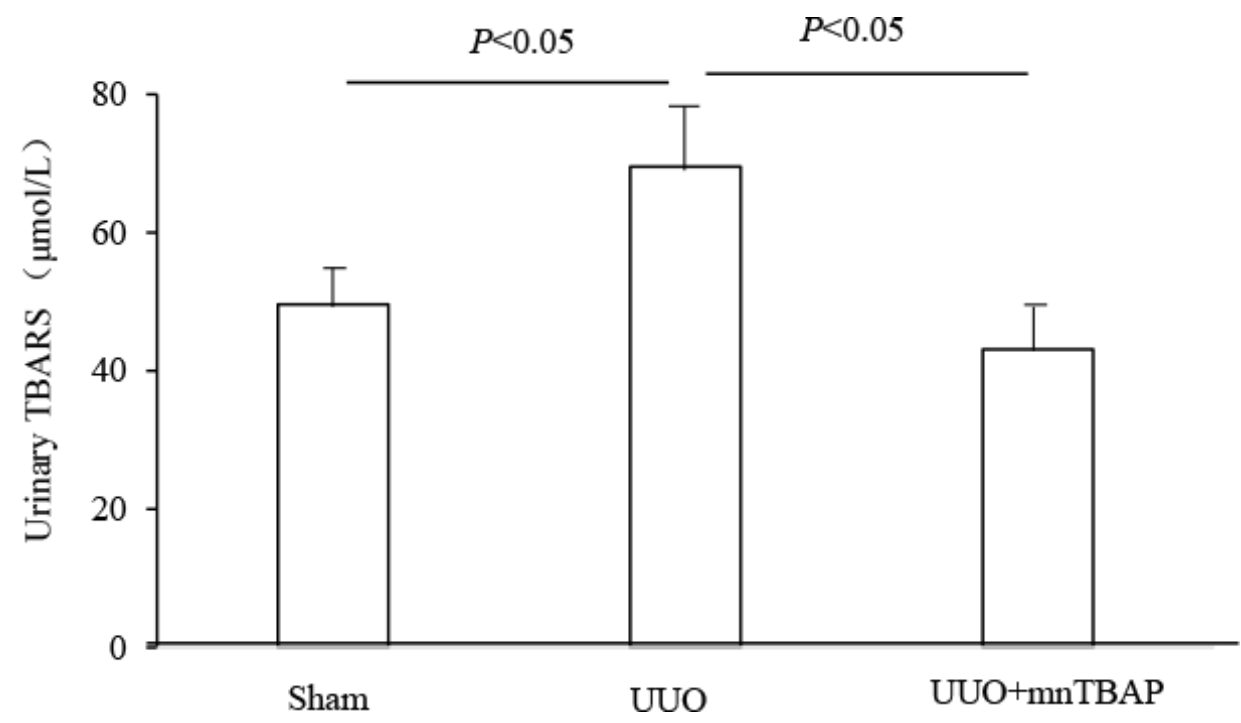

Figure 6: Urinary TBARS levels in UUO mice following MnTBAP treatment. The presented data are means \pm SE. $N=6$ in each group.
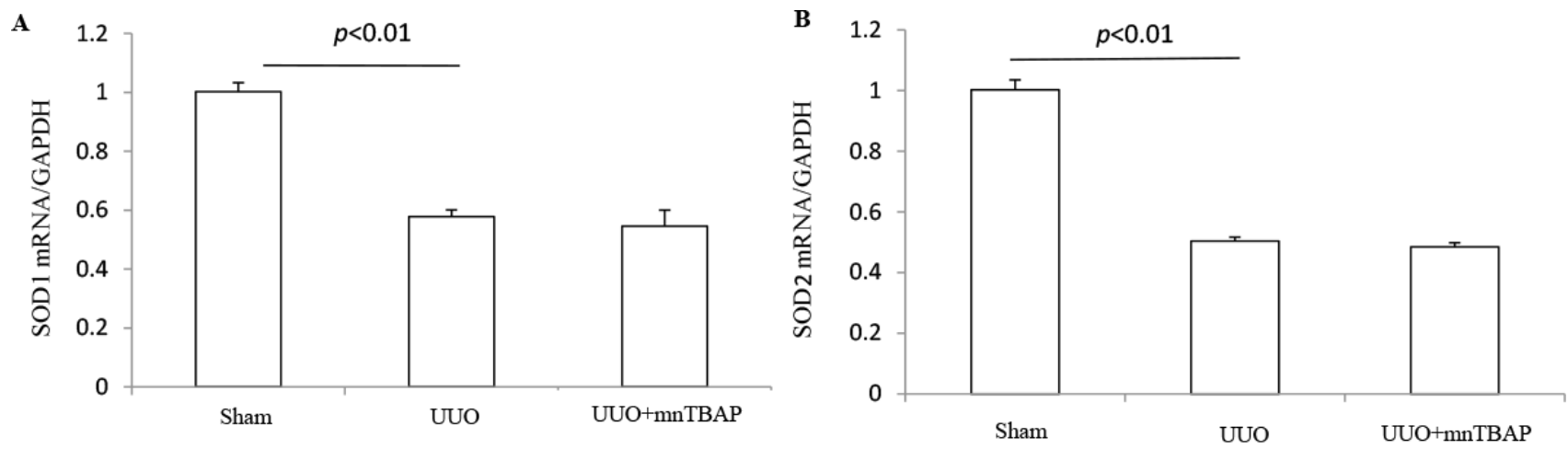

C

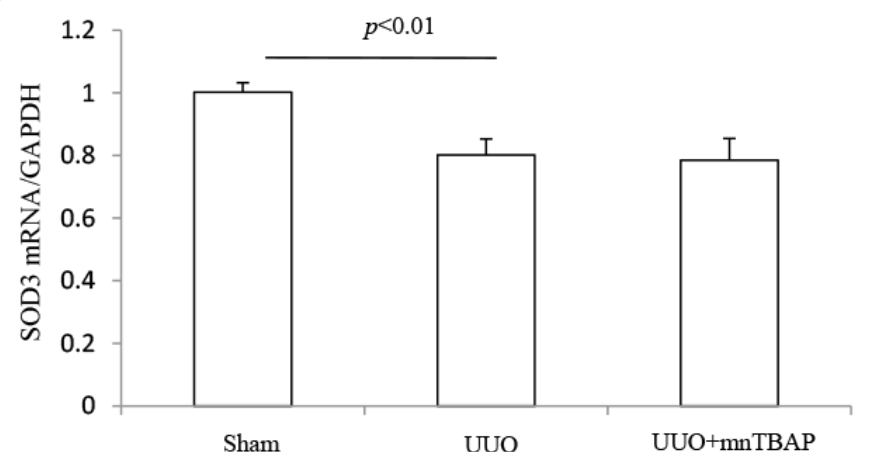

Figure 7: mRNA expressions of SOD1-3 in obstructed kidneys following MnTBAP treatment. (A) qRT-PCR analysis of SOD1. (B) qRT-PCR analysis of SOD2. (C) qRT-PCR analysis of SOD3. The presented data are means \pm SE. $N=6$ in each group. 
demonstrated that the mRNA levels of SOD1-3 were significantly diminished in obstructed kidneys. Hence, we administered a SOD mimetic compound, MnTBAP, to the mice after the surgery of UUO. Not surprisingly, the mice given MnTBAP exhibited partial but significant amelioration of the downregulation of NHE3, NCC, $\mathrm{NKCC} 2$. However, the downregulation of $\mathrm{ENaC} \alpha$ protein was not affected by MnTBAP therapy, suggesting that mitochondrial oxidative stress might not be important in modulating the sodium channels in collecting duct.

Another finding is that MnTBAP has impact only on the protein but not mRNA levels of NHE3, NCC, and NKCC2. This suggested that mitochondrial ROS may regulate these three transporters at posttranscriptional level. However, the exact mechanism mediating such an effect is still unknown. Then, we detected the expressions of SOD1-3 after kidney obstruction and MnTBAP administration. MnTBAP treatment normalized the oxidative stress marker TBARS in urine but did not affect the reduction of SOD1-3 during kidney obstruction, which might suggest that MnTBAP administration ameliorated oxidative stress during obstructive nephropathy without affecting the endogenous SOD.

Taken together, our study showed that the mitochondrial SOD mimics MnTBAP partially but significantly attenuated obstruction-induced downregulation of NHE3, NCC, and NKCC2 at protein levels in kidneys. The reduced mRNA levels of these transporters were not influenced by MnTBAP therapy. Meanwhile, MnTBAP did not affect the expressions of $\mathrm{ENaC}$ subunits in UUO model. These findings suggested that mitochondrial oxidative stress might mediate the downregulation of sodium transporters except $\mathrm{ENaC}$ in obstructive nephropathy via a posttranscriptional mechanism. This study not only provided new insights into the understanding of the pathogenesis of obstructive nephropathy-related dysregulation of sodium transporters but also offered new therapeutic target for the management of obstructive nephropathy-related fluid imbalance.

\section{MATERIALS AND METHODS}

\section{Animals}

C57BL/6J mice were originally purchased from the Jackson laboratory. This mouse colony was propagated at the Nanjing Medical University. In all studies, 3to 4-month-old male mice were used. All mice were maintained under a 12:12 h light-dark cycle (lights on at 6:00 a.m. and lights off at 6:00 p.m.). All experiments were approved by the Institutional Animal Care and Use Committee of Nanjing Medical University.

\section{Establishment of UUO mouse model and MnTBAP therapy}

Unilateral ureteral obstruction (UUO) was induced as described previously $[12,31,34]$. Briefly, the left ureter was exposed and subsequently ligated with 6.0 silk through a small abdominal incision under the anesthesia with $2.0 \%$ isoflurane. The abdomen was closed in two layers. All mice received analgesia (subcutaneous injection of $50 \mu \mathrm{g} / \mathrm{kg}$ buprenorphine (Temgesic, ScheringPlough)) after the surgery. Following the surgery, the UUO mice were treated with Manganese (III) tetrakis (4-benzoic acid) porphyrin chloride (MnTBAP) (Catalog No.: sc-221954A, Santa Cruz, Dallas, TA, USA), at a dose of $10 \mathrm{mg} / \mathrm{kg} /$ day in water by intraperitoneal injection. The control mice received sham operation and were treated with the same volume of water. All mice $(N=6$ per group) were sacrificed 7 days after operation and the kidney tissues were harvested for further analysis.

\section{Immunohistochemistry}

The kidneys were fixed in 10\% formalin and embedded in paraffin. Kidney sections (4- $\mu$ m thickness) were deparaffinized, hydrated in ethyl alcohol and washed in tap water. Endogenous peroxidase activity was blocked in $3 \% \mathrm{H}_{2} \mathrm{O}_{2}$ for $15 \mathrm{~min}$. Antigen retrieval was carried
Sham

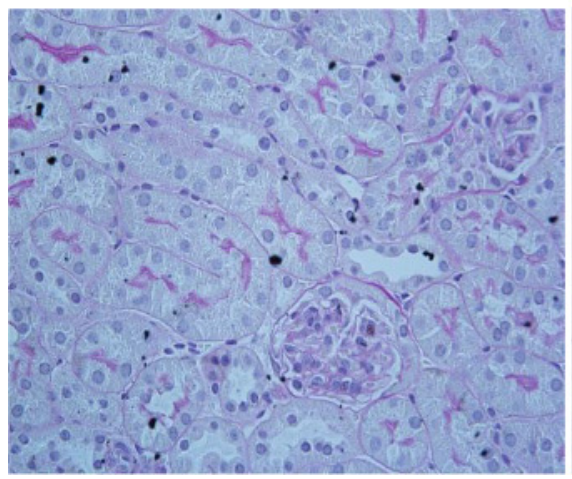

UUO

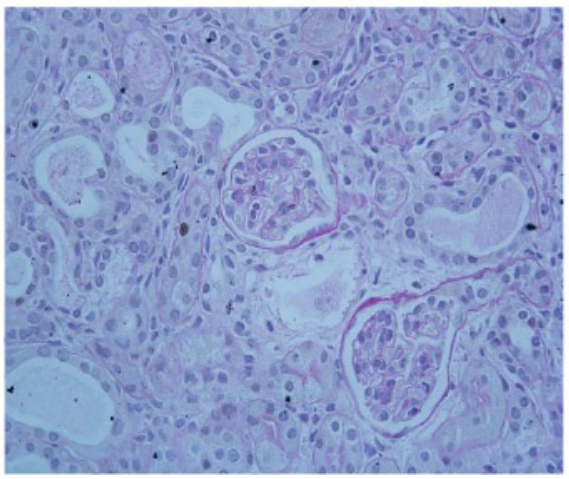

UUO+MnTBAP

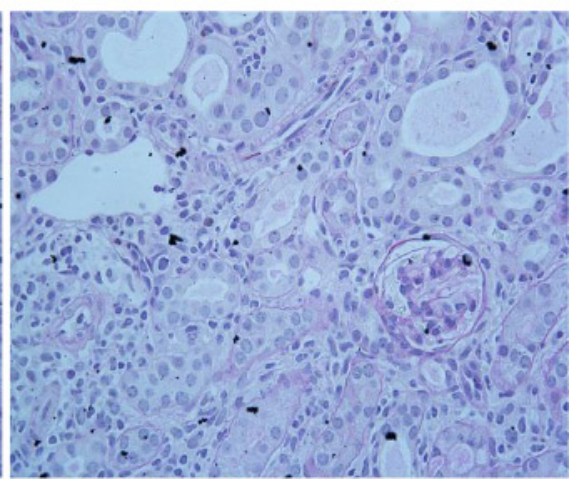

Figure 8: PAS staining in obstructed kidneys following MnTBAP treatment. 
Table 1: Sequences of qRT-PCR primers

\begin{tabular}{llc}
\hline Gene & \multicolumn{1}{c}{ Primer sequence } & Accession number \\
\hline GAPDH & 5'-GTCTTCACTACCATGGAGAAGG-3' & M32599 \\
ENaCa & 5'-TCATGGATGACCTTGGCCAG-3' & NM_011324 \\
& 5'-GCTTCATCTTTACCTGTCGTTTC-3' & \\
NCC & 5'-CCAGAGATTGGAGTTGTTCTTGT-3' & U61085 \\
& 5'-GACAGGCACCAACAGTGAGA-3' & \\
NKCC2 & 5'-TAGAGATGGCGGAGATGGAG-3' & NM_011389 \\
NHE3 & 5'-GCTCTTCATTCGCCTCTCCT-3' & \\
& 5'-AGCCTATTGACCCACCGAAC-3' & XM_993032 \\
SOD1 & 5'-CTGAGGAGGAACCGAGCA-3' & \\
& 5'-AGGCCCAGAACGATGAGTAG-3' & NM 921076 \\
SOD2 & 5'-AAGGCCGTGTGCGTGCTGAA-3' & \\
& 5'-CAGGTCTCCAACATGCCTCT-3' & NM 013671 \\
SOD3 & 5'-CGGCCTACGTGAACAATCTC-3' & \\
& 5'-GATAGCCTCCAGCAACTCTCC-3' & NM 011435 \\
\hline
\end{tabular}

out in boiling antigen retrieval solution $(1 \mathrm{mmol} / \mathrm{L}$ Tris$\mathrm{HCl}, 0.1 \mathrm{mmol} / \mathrm{L}$ EDTA, $\mathrm{pH}=8.0$ ) for 15 minutes. Then the sections were incubated overnight at $4^{\circ} \mathrm{C}$ with rabbit anti-NKCC2 antibody (Catalog No.: SPC-401, Stressmarq Biosciences Inc., Canada) and at room temperature for 30 min with secondary antibody. Staining was visualized using an $\mathrm{ABC}$ kit (Santa Cruz Biotechnology).

\section{Immunoblotting}

Proteins were extracted from the whole kidney and homogenized in protein lysis buffer. Equal amount of protein $(60 \mu \mathrm{g})$ was subjected to SDS-polyacrylamide gel electrophoresis, and transferred to nitrocellulose membranes. The blots were blocked at room temperature (RT) for $1 \mathrm{~h}$ with 5\% nonfat dry milk in Tris-buffered saline (TBS) and probed with the following antibodies: rabbit anti-NHE3 (Catalog No.: AB3085, Abcam, Cambridge, MA), antiNKCC2 (Catalog No.: SPC-401, Stressmarq Biosciences Inc., Canada), anti-NCC (Catalog No.: SPC-402, Stressmarq Biosciences Inc., Canada), anti-ENaC $\alpha$ (Catalog No.: SPC403, Stressmarq Biosciences Inc., Canada) and anti-ENaC $\gamma$ (Catalog No.: SPC-405, Stressmarq Biosciences Inc., Canada) at a dilution of $1: 1,000$ at $4^{\circ} \mathrm{C}$ overnight. After incubated with secondary antibodies (Catalog No.: sc-2004, goat anti-rabbit IgG, Santa Cruz) at RT for $1 \mathrm{~h}$, membranes were visualized using Pierce ${ }^{\mathrm{TM}}$ ECL Western Blotting Substrate (Thermo Scientific). Quantification was performed using Image-Pro Plus 6.0 software.

\section{Quantitative real-time PCR (qRT-PCR)}

Total RNA was isolated by using TRIzol (Invitrogen) and first-strand cDNA was synthesized from total RNA(4ug) using Superscript TM III first strand synthesis kit (Invitrogen). Quantitative PCR (qPCR) was performed using the SYBR Green Master Mix (Applied Biosystems, Warrington, UK) and the ABI 7500 Real-Time PCR Detection System (Applied Biosystems, Foster City). Oligonucleotides were designed using Primer3 software (available at http://frodo.wi.mit.edu/primer3/) and the sequences are listed in Table 1. Cycling conditions were $95^{\circ} \mathrm{C}$ for $10 \mathrm{~min}$, followed by 40 repeats of $95^{\circ} \mathrm{C}$ for $15 \mathrm{~s}$, and $60^{\circ} \mathrm{C}$ for $1 \mathrm{~min}$. Data were normalized to housekeeping gene GAPDH and presented as fold increase compared with RNA isolated from sham kidney using the $2{ }^{\Delta \Delta} \mathrm{CT}$ method.

\section{Measurement of TBARS}

The measurement of urinary thiobarbituric acidreactive substances (TBARS) was based on the formation of malondialdehyde by using a commercially available TBARS Assay Kit (Catalog No.: 10009055, Cayman Chemical) according to the manufacturer's instructions.

\section{Statistical analysis}

All results were presented as means $\pm \mathrm{SE}$. The statistical analysis was performed using ANOVA followed by Bonferroni's test with SPSS 13 statistical software. $p<0.05$ was considered significant.

\section{ACKNOWLEDGMENTS AND FUNDING}

This work was supported by Grants from the National Natural Science Foundation of China (nos. $81370802,81300591,81670647,81600557,81770690$, and 81570616), the National Key Research and Development Program (no. 2016YFC0906103), and the Natural Science Foundation of Jiangsu Province (no. BK2012001). 


\section{CONFLICTS OF INTEREST}

There is no conflicts of interest to disclose.

\section{REFERENCES}

1. Li C, Wang W, Kwon TH, Knepper MA, Nielsen S, Frokiaer J. Altered expression of major renal $\mathrm{Na}$ transporters in rats with unilateral ureteral obstruction. Am J Physiol Renal Physiol. 2003; 284: F155-66. https://doi. org/10.1152/ajprenal.00272.2002.

2. Jensen AM, Li C, Praetorius HA, Norregaard R, Frische S, Knepper MA, Nielsen S, Frokiaer J. Angiotensin II mediates downregulation of aquaporin water channels and key renal sodium transporters in response to urinary tract obstruction. Am J Physiol Renal Physiol. 2006; 291:F1021-32. https:// doi.org/10.1152/ajprenal.00387.2005.

3. Norregaard R, Jensen BL, Li C, Wang W, Knepper MA, Nielsen S, Frokiaer J. COX-2 inhibition prevents downregulation of key renal water and sodium transport proteins in response to bilateral ureteral obstruction. Am J Physiol Renal Physiol. 2005; 289:F322-33. https://doi. org/10.1152/ajprenal.00061.2005.

4. Kawada N, Moriyama T, Ando A, Fukunaga M, Miyata T, Kurokawa K, Imai E, Hori M. Increased oxidative stress in mouse kidneys with unilateral ureteral obstruction. Kidney Int. 1999; 56:1004-13. https://doi. org/10.1046/j.1523-1755.1999.00612.x.

5. Manucha W, Carrizo L, Ruete C, Molina H, Valles P. Angiotensin II type I antagonist on oxidative stress and heat shock protein 70 (HSP 70) expression in obstructive nephropathy. Cell Mol Biol (Noisy-le-grand). 2005; 51:547-55.

6. Dendooven A, Ishola DA Jr, Nguyen TQ, Van der Giezen DM, Kok RJ, Goldschmeding R, Joles JA. Oxidative stress in obstructive nephropathy. Int J Exp Pathol. 2011; 92:202-10. https://doi.org/10.1111/j.1365-2613.2010.00730.x.

7. Gill PS, Wilcox CS. NADPH oxidases in the kidney. Antioxid Redox Signal. 2006; 8:1597-607. https://doi.org/10.1089/ ars.2006.8.1597.

8. Sena LA, Chandel NS. Physiological roles of mitochondrial reactive oxygen species. Mol Cell. 2012; 48:158-67. https:// doi.org/10.1016/j.molcel.2012.09.025.

9. Jha JC, Banal C, Chow BS, Cooper ME, Jandeleit-Dahm K. Diabetes and Kidney Disease: Role of Oxidative Stress. Antioxid Redox Signal. 2016. https://doi.org/10.1089/ ars.2016.6664.

10. Liu C, Song Y, Qu L, Tang J, Meng L, Wang Y. Involvement of NOX in the regulation of renal tubular expression of $\mathrm{Na} / \mathrm{K}$ ATPase in acute unilateral ureteral obstruction rats. Nephron. 2015; 130:66-76. https://doi.org/10.1159/000381858.

11. Sun Y, Zhang Y, Zhu Y, Zhang A, Huang S, Yin X, Ding G, Liu M, Jia Z. Inhibition of Mitochondrial Complex-1 Restores the Downregulation of Aquaporins in Obstructive
Nephropathy. Am J Physiol Renal Physiol. 2016; 311:F777F786. https://doi.org/10.1152/ajprenal.00215.2015.

12. Zhang Y, Sun Y, Ding G, Huang S, Zhang A, Jia Z. Inhibition of Mitochondrial Complex-1 Prevents the Downregulation of NKCC2 and ENaCalpha in Obstructive Kidney Disease. Sci Rep. 2015; 5:12480. https://doi.org/10.1038/srep12480.

13. Kim SW, Lee J, Jung K, Ma SK, Oh Y, Kim WY, Choi KC, Kim J. Diminished expression of sodium transporters in the ureteral obstructed kidney in rats. Nephron Exp Nephrol. 2004; 96: e67-76. https://doi.org/10.1159/000076748.

14. Li C, Wang W, Kwon TH, Knepper MA, Nielsen S, Frokiaer J. Altered expression of major renal $\mathrm{Na}$ transporters in rats with bilateral ureteral obstruction and release of obstruction. Am J Physiol Renal Physiol. 2003; 285: F889901. https://doi.org/10.1152/ajprenal.00170.2003.

15. Wang W, Luo R, Lin Y, Wang F, Zheng P, Levi M, Yang T, Li C. Aliskiren restores renal AQP2 expression during unilateral ureteral obstruction by inhibiting the inflammasome. Am J Physiol Renal Physiol. 2015; 308:F910-22. https://doi.org/10.1152/ajprenal.00649.2014.

16. Zhang Y, Listhrop R, Ecelbarger CM, Kishore BK. Renal sodium transporter/channel expression and sodium excretion in $\mathrm{P} 2 \mathrm{Y} 2$ receptor knockout mice fed a high- $\mathrm{NaCl}$ diet with/without aldosterone infusion. Am J Physiol Renal Physiol. 2011; 300: F657-68. https://doi.org/10.1152/ ajprenal.00549.2010.

17. Wang W, Li C, Nejsum LN, Li H, Kim SW, Kwon TH, Jonassen TE, Knepper MA, Thomsen K, Frokiaer J, Nielsen S. Biphasic effects of ANP infusion in conscious, euvolumic rats: roles of AQP2 and ENaC trafficking. Am J Physiol Renal Physiol. 2006; 290: F530-41. https://doi. org/10.1152/ajprenal.00070.2005.

18. Kinter M, Wolstenholme JT, Thornhill BA, Newton EA, McCormick ML, Chevalier RL. Unilateral ureteral obstruction impairs renal antioxidant enzyme activation during sodium depletion. Kidney Int. 1999; 55:1327-34. https://doi.org/10.1046/j.1523-1755.1999.00358.x.

19. Manucha W, Valles PG. Apoptosis modulated by oxidative stress and inflammation during obstructive nephropathy. Inflamm Allergy Drug Targets. 2012; 11:303-12.

20. Omori H, Kawada N, Inoue K, Ueda Y, Yamamoto R, Matsui I, Kaimori J, Takabatake Y, Moriyama T, Isaka Y, Rakugi H. Use of xanthine oxidase inhibitor febuxostat inhibits renal interstitial inflammation and fibrosis in unilateral ureteral obstructive nephropathy. Clin Exp Nephrol. 2012; 16:549-56. https://doi.org/10.1007/s10157-012-0609-3.

21. Wang Y, Wang B, Du F, Su X, Sun G, Zhou G, Bian X, Liu N. Epigallocatechin-3-Gallate Attenuates Oxidative Stress and Inflammation in Obstructive Nephropathy via NF-kappaB and Nrf2/HO-1 Signalling Pathway Regulation. Basic Clin Pharmacol Toxicol. 2015; 117:164-72. https:// doi.org/10.1111/bcpt.12383.

22. Zecher M, Guichard C, Velasquez MJ, Figueroa G, Rodrigo R. Implications of oxidative stress in the 
pathophysiology of obstructive uropathy. Urol Res. 2009; 37:19-26. https://doi.org/10.1007/s00240-008-0163-3.

23. Brownlee M. Biochemistry and molecular cell biology of diabetic complications. Nature. 2001; 414:813-20. https:// doi.org/10.1038/414813a.

24. Wan $\mathrm{C}, \mathrm{Su} \mathrm{H}$, Zhang C. Role of NADPH Oxidase in Metabolic Disease-Related Renal Injury: An Update. Oxid Med Cell Longev. 2016; 2016: 7813072. https://doi. org/10.1155/2016/7813072.

25. Nie H, Xue X, Liu G, Guan G, Liu H, Sun L, Zhao L, Wang X, Chen Z. Nitro-oleic acid ameliorates oxygen and glucose deprivation/re-oxygenation triggered oxidative stress in renal tubular cells via activation of Nrf2 and suppression of NADPH oxidase. Free Radic Res. 2016; 50:1200-1213. https://doi.org/10.1080/10715762.2016.1225955.

26. Liu J, Wang C, Liu F, Lu Y, Cheng J. Metabonomics revealed xanthine oxidase-induced oxidative stress and inflammation in the pathogenesis of diabetic nephropathy. Anal Bioanal Chem. 2015; 407:2569-79. https://doi. org/10.1007/s00216-015-8481-0.

27. Munoz M, Sanchez A, Pilar Martinez M, Benedito S, Lopez-Oliva ME, Garcia-Sacristan A, Hernandez M, Prieto D. COX-2 is involved in vascular oxidative stress and endothelial dysfunction of renal interlobar arteries from obese Zucker rats. Free Radic Biol Med. 2015; 84:77-90. https://doi.org/10.1016/j.freeradbiomed.2015.03.024.

28. Satoh M, Fujimoto S, Haruna Y, Arakawa S, Horike H, Komai N, Sasaki T, Tsujioka K, Makino H, Kashihara N. $\mathrm{NAD}(\mathrm{P}) \mathrm{H}$ oxidase and uncoupled nitric oxide synthase are major sources of glomerular superoxide in rats with experimental diabetic nephropathy. Am J Physiol Renal Physiol. 2005; 288:F1144-52. https://doi.org/10.1152/ ajprenal.00221.2004.
29. Xu Y, Ruan S, Wu X, Chen H, Zheng K, Fu B. Autophagy and apoptosis in tubular cells following unilateral ureteral obstruction are associated with mitochondrial oxidative stress. Int J Mol Med. 2013; 31:628-36. https://doi. org/10.3892/ijmm.2013.1232.

30. Nishida H, Kurahashi T, Saito Y, Otsuki N, Kwon M, Ohtake H, Yamakawa M, Yamada KI, Miyata S, Tomita Y, Fujii J. Kidney fibrosis is independent of the amount of ascorbic acid in mice with unilateral ureteral obstruction. Free Radic Res. 2014; 48:1115-24. https://doi.org/10.3109 /10715762.2014.915031.

31. Sun Y, Zhang Y, Zhao D, Ding G, Huang S, Zhang A, Jia Z. Rotenone remarkably attenuates oxidative stress, inflammation, and fibrosis in chronic obstructive uropathy. Mediators Inflamm. 2014; 2014:670106. https://doi.org/ $10.1155 / 2014 / 670106$.

32. Bresciani G, da Cruz IB, Gonzalez-Gallego J. Manganese superoxide dismutase and oxidative stress modulation. Adv Clin Chem. 2015; 68:87-130. https://doi.org/10.1016/ bs.acc.2014.11.001.

33. Chuang ST, Kuo YH, Su MJ. KS370G, a caffeamide derivative, attenuates unilateral ureteral obstructioninduced renal fibrosis by the reduction of inflammation and oxidative stress in mice. Eur J Pharmacol. 2015; 750:1-7. https://doi.org/10.1016/j.ejphar.2015.01.020.

34. Rouschop KM, Sewnath ME, Claessen N, Roelofs JJ, Hoedemaeker I, van der Neut R, Aten J, Pals ST, Weening JJ, Florquin S. CD44 deficiency increases tubular damage but reduces renal fibrosis in obstructive nephropathy. J Am Soc Nephrol. 2004; 15:674-86. 